\title{
Genome-wide Identification and Characterization of the DELLA Subfamily in Prunus mume
}

\author{
Jiuxing Lu, Weiru Yang, and Qixiang Zhang ${ }^{1}$ \\ Beijing Key Laboratory of Ornamental Plants Germplasm Innovation \& Molecular Breeding, National \\ Engineering Research Center for Floriculture, Beijing Laboratory of Urban and Rural Ecological \\ Environment and College of Landscape Architecture, Beijing Forestry University, Beijing, 100083, China
}

\begin{abstract}
AdDitional INDEX wORDs. mei, bioinformatics, functional analysis, breeding, gibberellins
Abstract. Control of development is an important issue in the production of ornamental plants. Gibberellins (GAs) play a key role in regulating plant growth and development. DELLA is nuclear negative regulators of GA signaling. We identified two DELLA homologous genes, PmDELLA1 and PmDELLA2, in the genome of mei (Prunus mume) genome. We analyzed the structure, expression patterns and molecular functions of both genes. Tissue expression analysis showed that both genes were transcriptionally active. PmDELLA1 showed higher expression in seeds than PmDELLA2. This indicated that $P m D E L L A 2$ plays different roles from $P m D E L L A 1$ in seed germination. The expressions of both genes at various flowering stages were relatively low. We speculated that $P m D E L L A s$ might be positive regulators of flowering by releasing the repression of GA during floral blooming. Transgenic arabidopsis (Arabidopsis thaliana) lines overexpressing the two genes showed dwarf and delayed flowering. We confirmed that the two PmDELLAs were partially conserved with genes encoding DELLA proteins in arabidopsis. Our bioinformatics and functional analyses provide information that may be valuable to improve the economic, agronomic and ecological properties of mei and other Rosaceae fruit trees.
\end{abstract}

Gibberellins are phytohormones that promote important aspects of growth, such as seed germination, leaf expansion, stem elongation, flowering, and fruit development (Davies, 1995). In woody plants, many GA-mediated processes are agriculturally important. GA levels are routinely manipulated by chemical means to produce desirable effects in a number of species (Looney, 1997; Rademacher, 2000). For example, exogenous GA is used to control flowering time and increase fruit size, and inhibitors of GA biosynthesis are applied to restrict vegetative growth. Mei, domesticated in China for more than 3000 years, is one of the most important ornamental flower and fruit trees among the Rosaceae. In East Asia, the flower and young fruit of mei can tolerate low temperatures $\left(-4\right.$ to $\left.-2{ }^{\circ} \mathrm{C}\right)$ in early spring (Chen, 1996). Mei blooms in late winter or early spring (typically late January or February) before the new leaves grow. Its special fragrance makes it popular with Chinese people (Chen, 1996). However, it is too big in size for us to put common mei indoors. We need to minimize the size of mei to meet customer demands. In addition, regulation of flowering time is also key problems for us. So, it is necessary to investigate the functions of GA-associated transcriptional regulators, such as DELLA, in mei.

DELLA is a key component of the GA signaling pathway, acting as negative elements. They constitute a subgroup of the GRAS gene family (whose name was derived from the first three functionally characterized genes, GAI, RGA, and $S C R$ ), which encode plant-specific transcription factors. DELLA was named from a short stretch of amino acids (D-E-L-L-A) in their $\mathrm{N}$-terminal region, which is highly conserved among plant species (Locascio et al., 2013) (Fig. 1A). They also have

Received for publication 15 Oct. 2014. Accepted for publication 4 Mar. 2015. The research was supported by Ministry of Science and Technology (grants 2011AA100207 and 2013AA102607) and Special Fund for Beijing Common Construction Project.

${ }^{1}$ Corresponding author. E-mail: zqxbjfu@126.com. additional conserved motifs, such as the TVHYNP domain; a poly(serine/threonine) stretch; two leucine heptad repeats, which may mediate protein-protein interactions; a putative nuclear localization signal; and a putative SH2 phosphotyrosinebinding domain (Locascio et al., 2013) (Fig. 1A). Mutants lacking DELLA motif show a phenotype similar to that of continuous gibberellin application (Cao et al., 2005; Cheng et al., 2004; Dill and Sun, 2001; Tyler et al., 2004). For example, the stable mutants gai-1 and rga-17 show a dwarf phenotype in arabidopsis equivalent to that caused by gibberellin deficiency (Dill and Sun, 2001; Peng et al., 1997). Similar functions of DELLA were found in rice (Oryza sativa) (SLR1, DLT), wheat (Triticum aestivum) (RhtBlb, RhtDlb), maize (Zea mays) (D8), barley (Hordeum vulgare) (SLN1), and grape (Vitis vinifera) (VvGAI) (Boss and Thomas, 2002; Chandler et al., 2002; Ogawa et al., 2000; Peng et al., 1999). For example, the semidominant dwarf wheat mutants that were the basis of the 'Green Revolution' wheat are caused by a mutation in the DELLA region of the Rht-Bl and Rht-Dl genes (Ogawa et al., 2000; Peng et al., 1999). The highly productive, semidwarfed 'Pinot Meunoir' grape contains a single amino acid change in the DELLA domain of $V v G A I$ (Boss and Thomas, 2002; Chandler et al., 2002). In many species, mutations of the DELLA motif are highly conserved (Dill and Sun, 2001; Itoh et al., 2002). There are five genes (GAI, RGA, RGL1, RGL2, and $R G L 3$ ) encoding DELLA protein in arabidopsis (Dill and Sun, 2001; Lee et al., 2002; Silverstone et al., 1998). Phenotypic analysis of the five proteins revealed their unique roles in the plant, although with a certain degree of overlapping functions. For example, $G A I$ and $R G A$ function to control cell expansion in hypocotyls, shoots and roots, while RGL2 is the major DELLA regulating germination (Lee et al., 2002; Tyler et al., 2004; Wen and Chang, 2002). Recent research speculated that the functional diversification of the different DELLA genes in arabidopsis probably reflect changes in their regulatory sequences (Gallego-Bartolomé et al., 2010). 
A

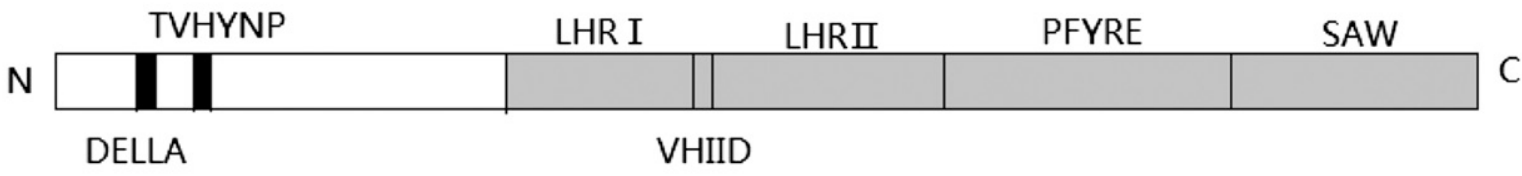

B

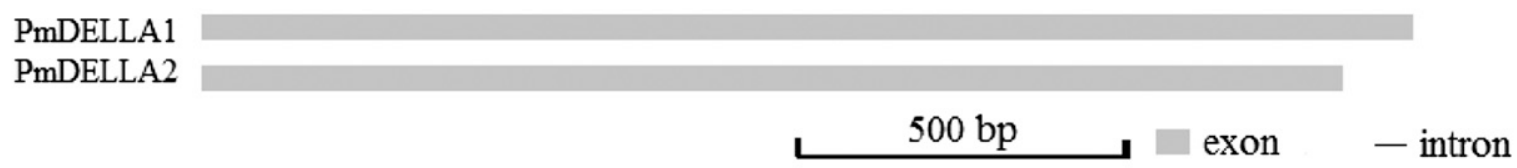

C

DELLA

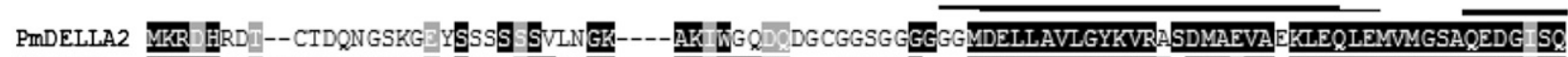

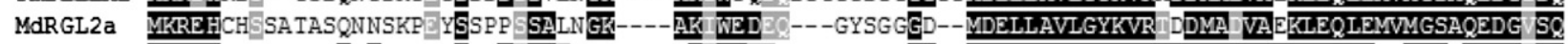

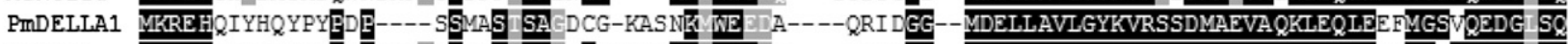

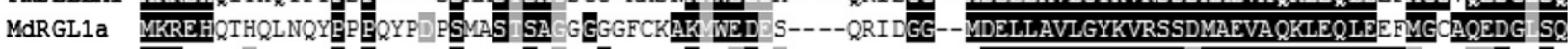
RGL2 MKRGYGE TVHYNP

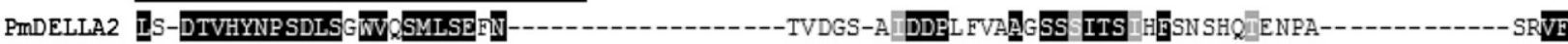

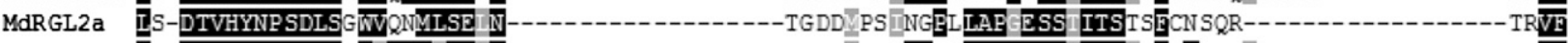

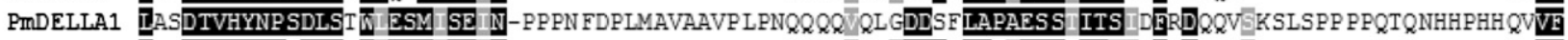

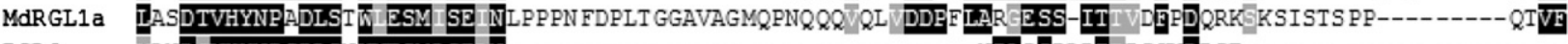

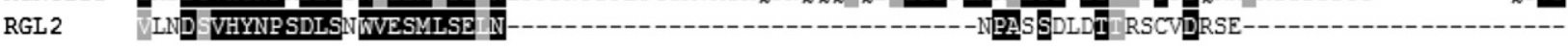

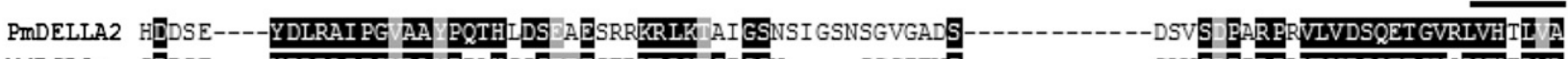

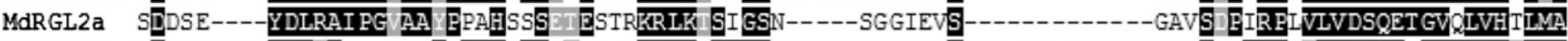

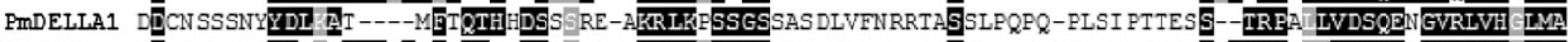

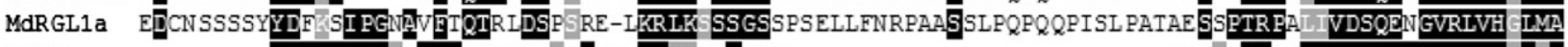

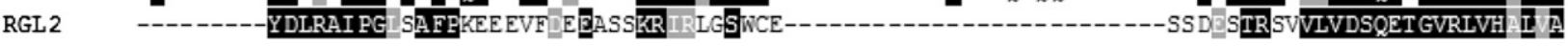
LHR I

PMDELLA2 CAEAVQQENL/LADALVK VGLLA SQAGAMRKVATYFAEALARRI YR YPQD----S DSS SD LQMHFYETCPYLKFAHFTANQAILEA FATI MdRGL2a CAEAVQQENL KLADALVK VGILA AQRTGAMRKVATYFAEALARRI YR YPQ ----CIDSS SD LQMHFYETCPYLKEAHFTANQAILEA EATA

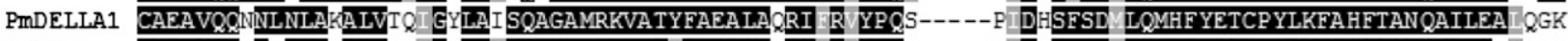

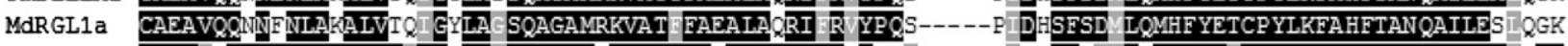

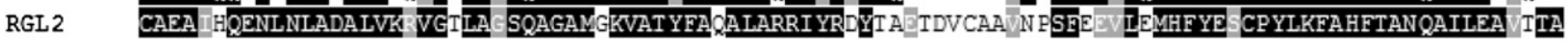
VHIID LHR II

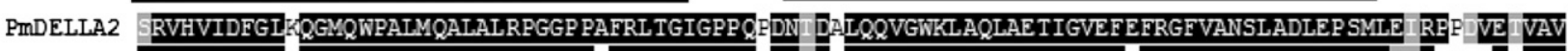
MdRGL2a TRVHVIDFGIKQGMQWPALMQALALRPGGP PVERLTGIGPPQ DDN DVLQQVGWKLAQLAETIGVEF GERGFVANSLADLEP SMID RQD-- EAVAV PMDELLA1 TRVHVIDES WQGMQWPALMQALALRPGGPPAFRLTGIGPFA DISSDHLQE VGWKLAQLAETIHVEFE RGFVANSLADI A SMLE RES VE VAV

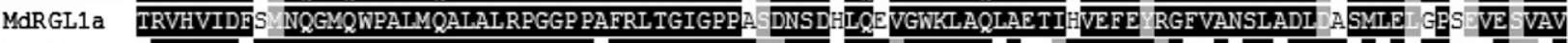

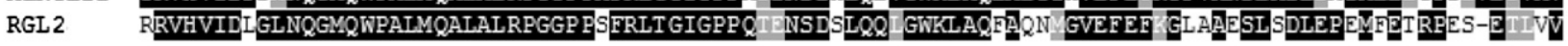
PFYRE

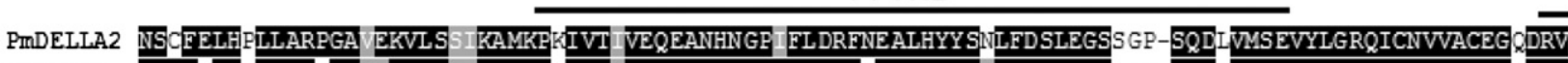
MdRGL2a WSVFKLHG GLARAGA KVLS KAMKEKIVI VEQEANHINGPVFLDRETEALHYYS LFDSLEGSSGP-SQDIVMSEVYLGRQICNVVACEGGDRV

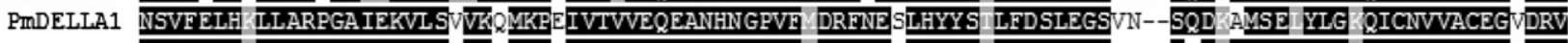

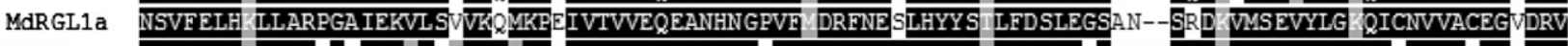
RGL2 WSVEELH LLARSGSIEK L WKA KR IVTVVEQEANHWGIVFLDRENEALHYYS LFDSLEDGY SLPSQD WWSEVYLGRQILVVVA AEGSDRV SAW

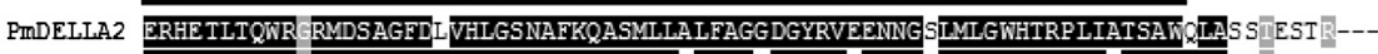

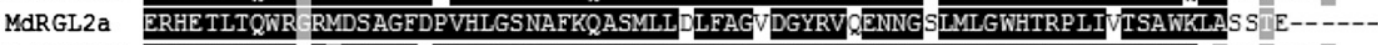

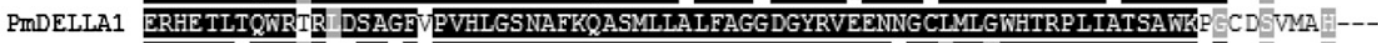

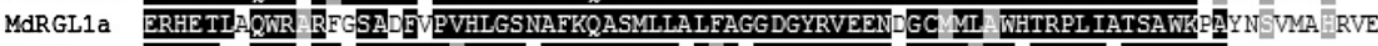

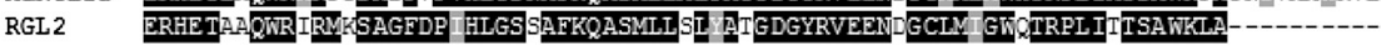

Fig. 1. (A) Key domains structure map of DELLA proteins. (B) Gene structure maps of PmDELLA1 and PmDELLA2. (C) Multiple alignments results of the deduced amino sequences of DELLA in Prunus mume (PmDELLA1 and PmDELLA2), Malus $\times$ domestica (MdRGL1a, MdRGL2a), and Arabidopsis thaliana (RGL2) are shown. Gray blocks: key domains of the GRAS protein family. Black blocks indicate DELLA subfamily-specific domains. Overhead lines indicate conserved motifs. Dashes indicate gaps to maximize alignment. 
In this study, two genes likely encoding DELLA proteins (PmDELLA1 and PmDELLA2) were identified from the mei genome database. They were cloned and their gene expression patterns and the effects of their transformation were observed. We aimed 1) to identify and characterize PmDELLAs in mei; 2) to analyze deduced amino acid sequences of PmDELLAs relationships among plants through comparative genomics; 3 ) to analyze their expression patterns in mei; 4) to perform functional analysis of PmDELLAs in transgenic arabidopsis; and 5) to provide useful information for further breeding of mei, such as that related to flowering time and plant size.

\section{Materials and Methods}

Sequence Retrieval. The genome files were downloaded from the mei genome project (Zhang et al., 2012b). Using the five DELLA protein sequences from arabidopsis as queries, the mei genomic database was blasted using local blast software. Default parameters were used in the analyses, and false hits were removed by manual inspection. All obtained protein sequences were subjected to domain analysis using protein sequence analysis and classification software [InterProScan (Jones et al., 2014)] to detect the presence of the DELLA domain, using default parameters. Protein sequences lacking the DELLA domain were rejected. The physicochemical properties of the identified protein sequences were predicted by the expert protein analysis system server (Artimo et al., 2012). Information on previously reported DELLA proteins of arabidopsis was retrieved from the arabidopsis information resource (Rhee et al., 2003). The genome data of apple (Malus $\times$ domestica), strawberry (Fragaria vesca), cucumber (Cucumis sativus), barrel medic (Medicago truncatula), black cottonwood (Populus trichocarpa), chinese cabbage (Brassica rapa), orange (Citrus sinensis), cocoa (Theobroma cacao), grape, tomato (Solanum lycopersicum), rice, maize, spikemoss (Selaginella moellendorffi), bryophyte (Physcomitrella patens), green alga (Chlamydomonas reinhardtii), red alga (Cyanidioschyzon merolae), and yeast (Saccharomyces cerevisiae) were collected from plant genome database [Phytozome (Goodstein et al., 2012)]. Genome data on banana [Musa acuinata (D'Hont et al., 2012)] were also collected. We used an online blast tool to identify DELLA protein sequences, using default parameters and manual removal of false hits. The protein family database [Pfam (Finn et al., 2013)] was used to identify the DELLA proteins sequences.

Multiple alignment analyses. Multiple sequence alignment of the full-length DELLA protein sequences of different species was performed using the ClustalX 2.1 program with its default settings (Larkin et al., 2007). Phylogenetic trees were constructed using the neighbor-joining method in the MEGA5.1 software (Tamura et al., 2011). Forty-one protein sequences were downloaded from the national center for biotechnology information website (NCBI) (Supplemental Table 1). We tested the reliability of the obtained tree using bootstrapping with 1000 replicates.

MRNA SEQUENCING AND ANALYSIS. We conducted gene expression analysis using RNA sequencing (RNA-seq) technology (Illumina, San Diego, CA). Five organs were obtained from 'Zangmei' mei in Tibet, China. Roots were harvested from 2-year-old plants of 'Zangmei' mei. Stems were obtained from a young stem tip. We collected new-sprouting young leaves in the spring. Fruit were obtained after 5 weeks of blooming. Organs were collected and immediately stored in liquid nitrogen.

Total RNA was isolated from the plant organs using an Easyspin $^{\text {TM }}$ kit (Aidlab, Beijing, China), following the instructions of the manufacturer. The sequencing and assembly were performed by the Beijing Genomics Institute (Beijing, China).

Gene Cloning. Young leaves of 'Yu die' mei from Jiufeng international plum blossom garden (Beijing, China), were collected and placed immediately in liquid nitrogen for subsequent cloning of the full-length cDNAs of PmDELLA1 and PmDELLA2. Gene-specific primers (Supplemental Table 2) were designed based on identified gene sequences from genome data. The full-length cDNAs were amplified using the following program: one cycle at $94{ }^{\circ} \mathrm{C}$ for $5 \mathrm{~min} ; 30$ cycles at $98^{\circ} \mathrm{C}$ for $5 \mathrm{~s}$, at $60^{\circ} \mathrm{C}$ for $15 \mathrm{~s}$, at $72^{\circ} \mathrm{C}$ for $1 \mathrm{~min} 30 \mathrm{~s}$; and hold at $72{ }^{\circ} \mathrm{C}$ for $10 \mathrm{~min}$. The resulting PCR product was separated on a $1 \%$ agarose gel and extracted using a BIOMIGAgel Purification Kit (Biomiga, San Diego, CA). Purified fragments were then cloned into the pGEM T-easy vector (Takara Biotechnology, Otsu, Japan) and transformed into Escherichia coli Top 10 cells (Tiangen, Beijing, China). Positive colons were confirmed by PCR analysis and sequencing of the inserted DNA (Taihe, Beijing, China).

Quantitative PCR. The expressions of PmDELLAs in different organs and at different developmental stages were examined using quantitative PCR. We extracted total RNA from roots, stems, leaves, seeds, flowers at three developmental stages (Flower A, B, and C; corresponding to bud, full-bloom stage, and end-bloom stages, respectively), and fruit at three developmental stages (Fruit A, B, and C; corresponding to 10, 45, and $90 \mathrm{~d}$ after blooming, respectively) using the TRIzol reagent (Invitrogen, Carlsbad, CA) following the instruction of the manufacturer. First-strand cDNA was synthesized from $2 \mu \mathrm{g}$ total RNA using the TIANScript Frist Strand cDNA Synthesis Kit (Tiangen), according to the instruction of the manufacturer. Quantitative PCR was performed using the PikoReal real-time PCR system (Thermo Fisher Scientific, Waltham, MA). Reactions were performed in a $20-\mu \mathrm{L}$ volume containing $1 \mu \mathrm{L}$ of cDNA, $300 \mathrm{nM}$ of each primer (Supplemental Table 2) and $10 \mu \mathrm{L}$ of SYBR Premix Ex Taq II (Takara Biotechnology). The reactions were performed under the following conditions: $30 \mathrm{~s}$ at $95{ }^{\circ} \mathrm{C}$; followed by 40 cycles of $5 \mathrm{~s}$ at $95^{\circ} \mathrm{C}$ and $30 \mathrm{~s}$ at $60{ }^{\circ} \mathrm{C}$.

The relative quantification values were calculated using a combination of two reference genes, the protein phosphatase 2A gene (PP2A), and ubiquitin-conjugating enzyme E2 (UBC). According previous study of the selection of reference genes in mei, PP2A and UBC were stably expressed in different organs and genotypes in mei (Wang et al., 2014b). The modified mathematical model was used to calculate the relative quantification (Wang et al., 2014a). The amplification efficiency of primer pairs of target genes and reference genes were derived from a standard curve generated from a serial dilution of the mixed cDNA from all tested samples as the template performed for each candidate reference gene in triplicate. Normalization factor for the two reference genes were calculated by geNorm V. 3.5 software (Vandesompele et al., 2002). There were three replicates of each organ and development stage.

Plant transformation. The full-length PmDELLA 1 and PmDELLA2 cDNAs were cloned separately into the pCAMBIA1304 plant transformation vector, containing the CaMV35S promoter, octopine synthase terminator, and 
Table 1. Identity of PmDELLA1 and PmDELLA2 from a local blast similarity search against Prunus mume genome and predicted physicochemical properties of deduced amino acid sequences of PmDELLA1 and PmDELLA2.

\begin{tabular}{|c|c|c|c|c|c|c|c|c|c|c|c|c|c|}
\hline & & & & mum & e gene ${ }^{z}$ & & & & & & & & \\
\hline$\overline{\text { Gene }}$ & & Length & MW & & & & Position & DELLA & $\overline{\text { DELLA }}$ & & lery gene & Resu & \\
\hline identity & Name & (aa) & $(\mathrm{kDa})$ & $\mathrm{P} I$ & GRAVY & Chr & $(\mathrm{Mbp})$ & motif start & motif end & Name & GenBank no. & Identity (\%) & $E$-value \\
\hline Pm006304 & PmDELLA1 & 633 & 70.0 & 5.33 & -0.316 & A2 & 16.0 & 52 & 124 & GAI & AT1G14920 & 74 & 0.0 \\
\hline & & & & & & & & & & RGL1 & AT1G66350 & 69 & 0.0 \\
\hline & & & & & & & & & & RGL2 & AT3G03450 & 66 & 0.0 \\
\hline & & & & & & & & & & RGL3 & AT5G17490 & 66 & 0.0 \\
\hline & & & & & & & & & & RGA & AT2G01570 & 63 & 0.0 \\
\hline & & & & & & & & & & RGL1 & AT1G66350 & 64 & 0.0 \\
\hline & & & & & & & & & & RGL2 & AT3G03450 & 61 & 0.0 \\
\hline & & & & & & & & & & RGL3 & AT5G17490 & 61 & 0.0 \\
\hline
\end{tabular}

${ }_{\mathrm{z}} \mathrm{aa}=$ amino acid; MW = molecular weight; $\mathrm{P} I=$ isoelectric point; GRAVY = grand average of hydropathy; Chr = chromosome number.

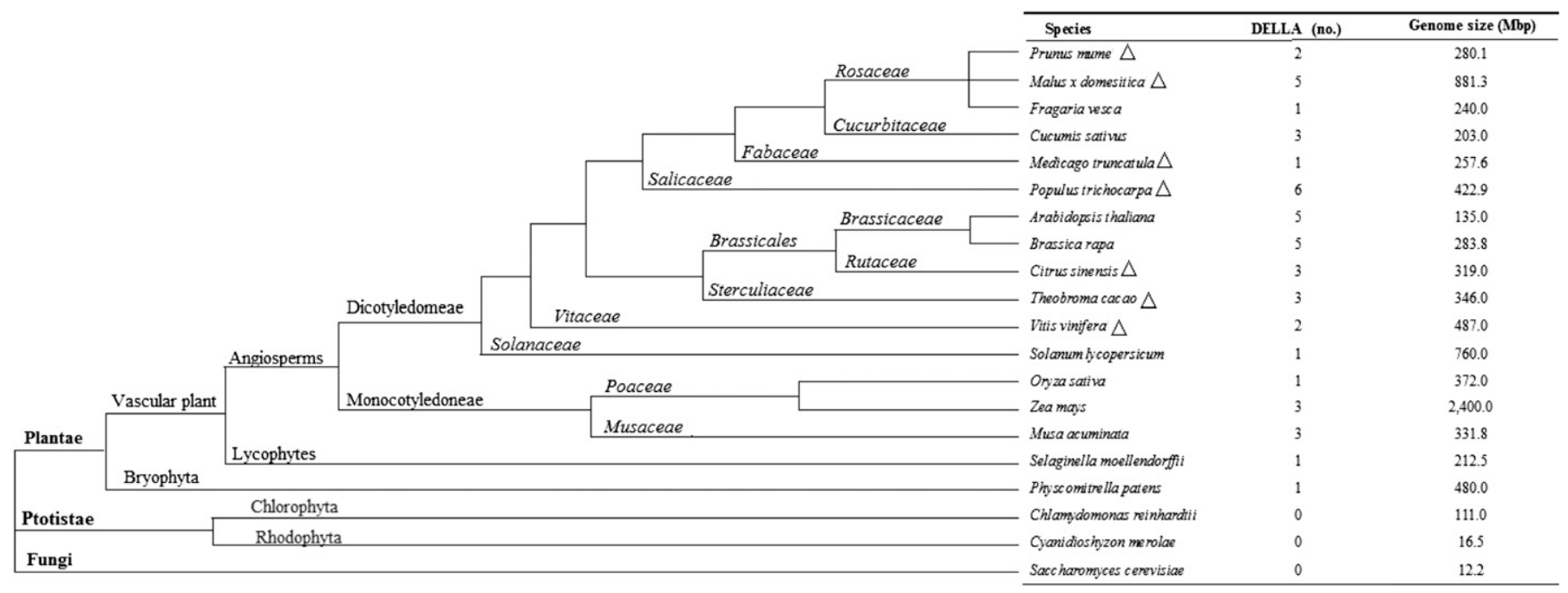

Fig. 2. Summary of the numbers of DELLA proteins and genome sizes of 20 representative species. The tree was constructed according to five kingdom classification system. The genome information of the 20 species, which represent the major evolutionary lineages of Prunus species, was acquired from plant genome database. Online blast tool of protein family database were used to identify DELLA proteins. Triangles indicate woody plants.

a hygromycin resistance gene as the selection marker. Agrobacterium (Agrobacterium tumifaciens) strain EHA105 was used for floral dip transformation of arabidopsis [Columbia ecotype (Clough and Bent, 1998)]. Resultant seeds were plated on Murashige and Skoog (MS) culture medium containing $30 \mu \mathrm{g} \cdot \mathrm{mL}^{-1}$ hygromycin to select for transformants. Total RNA was extracted from the leaves of mature plants and transgene expression levels were analyzed by quantitative PCR, using specific primers (Supplemental Table 1). Phenotypic analyses were performed on transgenic lines of $\mathrm{T}_{3}$ and $\mathrm{T}_{4}$ (third and fourth generations self-pollinated) with wild-type arabidopsis as the control (CK). For the analysis of leaf size and plant height, transformed $\mathrm{T}_{3}$ seedlings and $\mathrm{CK}$ were grown for $25 \mathrm{~d}$ in long-

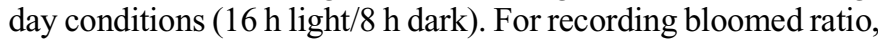
$\mathrm{T}_{3}$ seedlings and CK were grown for $60 \mathrm{~d}$ in short-day condition

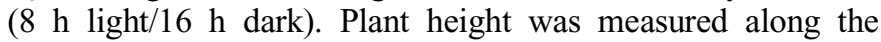
primary inflorescence axis and flowering was scored when petals were visible. For each treatment (long-day condition and shortday condition), we conducted in three repetitions. Data are collected from 20 plants per line for each repetition.

Seeds $\left(\mathrm{T}_{4}\right)$ from transgenic lines and CK were vernalized at $4{ }^{\circ} \mathrm{C}$ for $4 \mathrm{~d}$, sterilized, rinsed, and then plated onto $0.5 \times \mathrm{MS}$ medium or media containing $10^{-4} \mathrm{M} \mathrm{GA}_{3}$ (Biomiga). Seedlings were grown in a growth room maintained at $20^{\circ} \mathrm{C}$ with a $16-\mathrm{h}$ light/8-h dark cycle using fluorescent light for $7 \mathrm{~d}$. Seedlings were placed in a drop of water on a glass slide and the hypocotyl lengths (from the cotyledon shoulders to the collet of the root hairs) were measured from calibrated digital images (S8AP0 stereomicroscope with DFC500 camera; Leica, Wetzlar, Germany). The seedlings were measured for 25-30 plants per line for each repetition. This experiment was performed three times with similar results.

\section{Results}

Identification and characterization of PmDELLAs in MEI. Two genes encoding DELLA proteins from the entire mei genome were identified (Table 1). We named the two genes PmDELLA1 and PmDELLA2, and deduced amino sequences PmDELLA1 and PmDELLA2. The predicted PmDELLA1 and PmDELLA2 ORFs were 1899 and 1791 bp, respectively. Alignment with arabidopsis DELLA protein sequences showed that PmDELLA1 and PmDELLA2 were $61 \%$ to $74 \%$ homologous to the five arabidopsis DELLA proteins. Alignment 


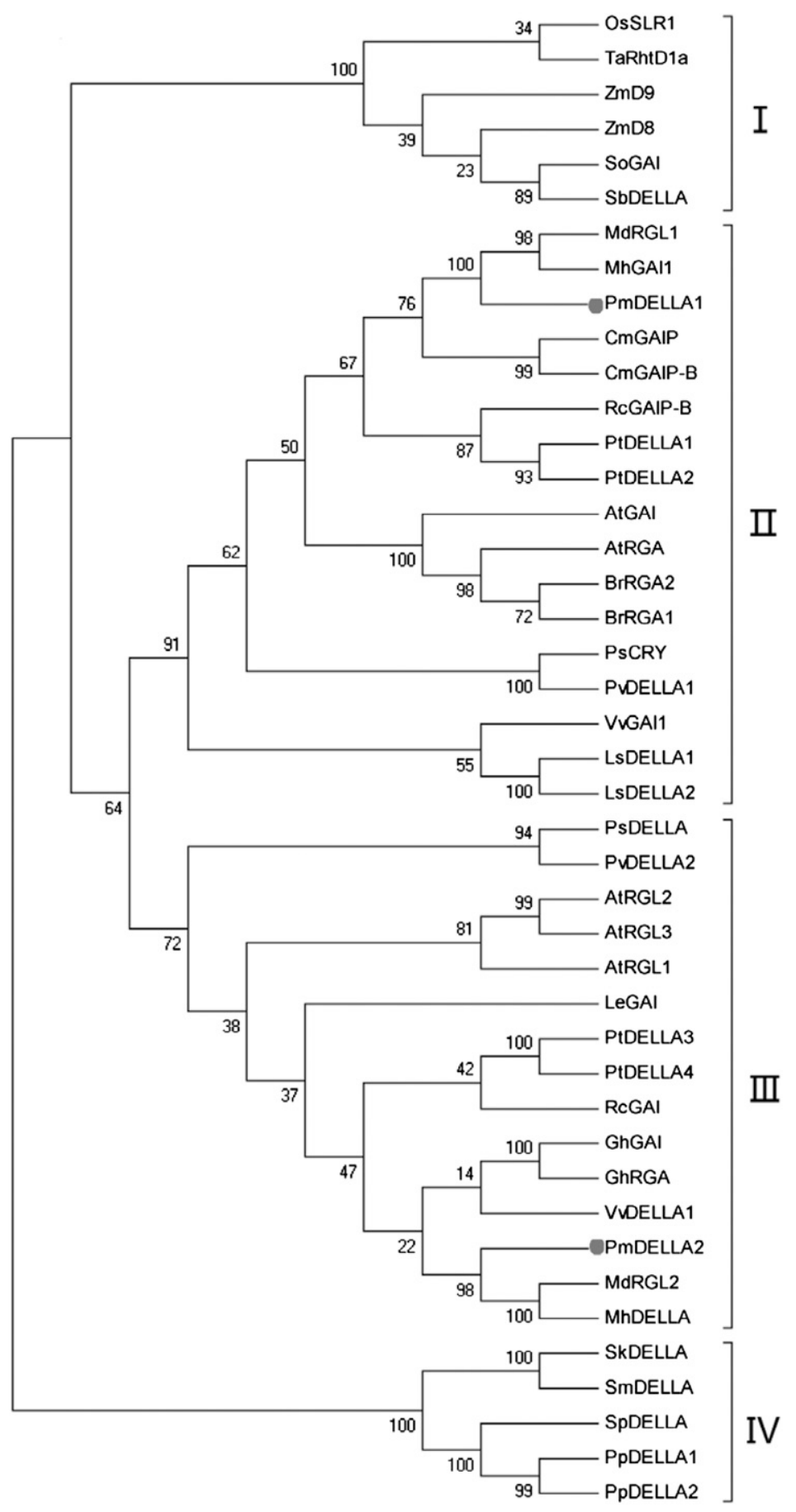

Fig. 3. Neighbor-joining distance tree showing similarity of DELLA protein sequences from Prunus mume (PmDELLA1 and PmDELLA2), Arabidopsis thaliana [AtGAI (CAA75492), AtRGA (CAA72177), AtRGL1 (NP_176809), AtRGL2 (NP_186995), AtRGL3 (NP_197251)], Brassica rapa [BrRGA2 (Q5BN23), BrRGA1 (Q5BN22)], Cucurbita maxima [CmGAIP (Q6EI06), CmGAIP-B (Q6EI05)], Gossypium hirsutum [GhGAI (Q84TQ7), GhRGA (AAY28970)], Lactuca sativa [LsDELLA1 (BAG71200), LsDELLA2 (BAG71201)], Lycopersicon esculentum [LeGAI (Q7Y1B6)], Malus $\times$ domestica [MdRGL1 (AAY56752), MdRGL2 (AAY56750)], Malus hupehensis [MhDELLA (ABS50250), MhGAI1 (ABL61270)], Oryza sativa [OsSLR1 (NP_001051032)], Phaseolus vulgaris [PvDELLA1 (BAF62636), PvDELLA2 (BAF62637)], Physcomitrella patens [PpDELLA1 (XM_001774262), PpDELLA2 (XM_001754038)], Pisum sativum [PsCRY (ABI34432), PsDELLA (ABI30654)], Populus trichocarpa [PtDELLA1 (XM_002312414), PtDELLA2 (XM_002314763), PtDELLA3 (XM_001774262), PtDELLA4 (XM_001754038)], Ricinus communis [RcGAI (XP_002534030), RcGAIP-B (XP_002527794)], Saccharum officinarum [SoGAI (AAZ08571)], Selaginella kraussiana [SkDELLA (ABU63412)], Selaginella moellendorffii [SmDELLA (ABX10758)], Sorghum bicolor [SbDELLA (XP_002466594)], Sphagnum palustre [SpDELLA (ABU63411)], Triticum aestivum [TaRhtD1a (Q9ST59)], Vitis vinifera [VvDELLA1 (XM_002534030), VvGAI1 (XM_002284612)], and Zea mays [ZmD8 (Q9ST48), ZmD9 (ABI84225)]. Numbers at nodes indicate bootstrap values (1000 replicates). Red dots indicate PmDELLA1 and PmDELLA2. of PmDELLA1 with PmDELLA2 showed that they shared $64 \%$ identity. The grand average of hydropathy showed that both proteins had a negative value, indicating that they were hydrophilic. In addition, PmDELLAs contain no introns in mei, which is consistent with counterparts from other plants (Fig. 1B) (Foster et al., 2007; Tian et al., 2004).

The predicted molecular weights of the two proteins were 64.7 and $70.0 \mathrm{kDa}$, respectively. PmDELLAs had two signature motifs, DELLA and TVHYNP, which define the DELLA subfamily and are necessary for GA-induced degradation of DELLA proteins (Fig. 1C). The C-termini of the PmDELLAs had five highly conserved motifs (LHR I, VHIID, LHR II, PFYRE, and SAW), which are shared by GRAS proteins. The PmDELLAs were most divergent in their N-termini, and highly homologous over their C-termini. This finding was consistent with previous studies (Foster et al., 2007; Pysh et al., 1999).

COMPARATIVE AND PHYLOGENETIC ANALYSES OF PMDELLAs IN DIFFERENT SPECIES. For comparative genomic analysis, we searched the genomes of 20 species (Fig. 2), and identified 48 DELLA protein sequences. These species represent the major evolutionary lineages of Prunus species and were used for the analysis of evolution of DELLA subfamily. Among the 20 species, only 17 species found DELLA proteins, whereas the two protists (green alga and red alga) and one fungus (yeast) searched no DELLA proteins. This observation indicated that DELLA may have expanded after the divergence of the plants from protest and fungi and that these proteins may have important roles in the evolution of plants. This result was consistent with the evolution of GRAS proteins (Song et al., 2014). The previous study showed that the GRAS family belongs to the Rossmann fold methyltransferase superfamily, which first emerged in bacteria; however, most GRAS proteins are likely to lack methyltransferase activity. Nevertheless, the plant GRAS proteins still bind a similar substrate to the bacterial proteins (Zhang et al., 2012a). We believed DELLA subfamily were a group of archaic proteins 

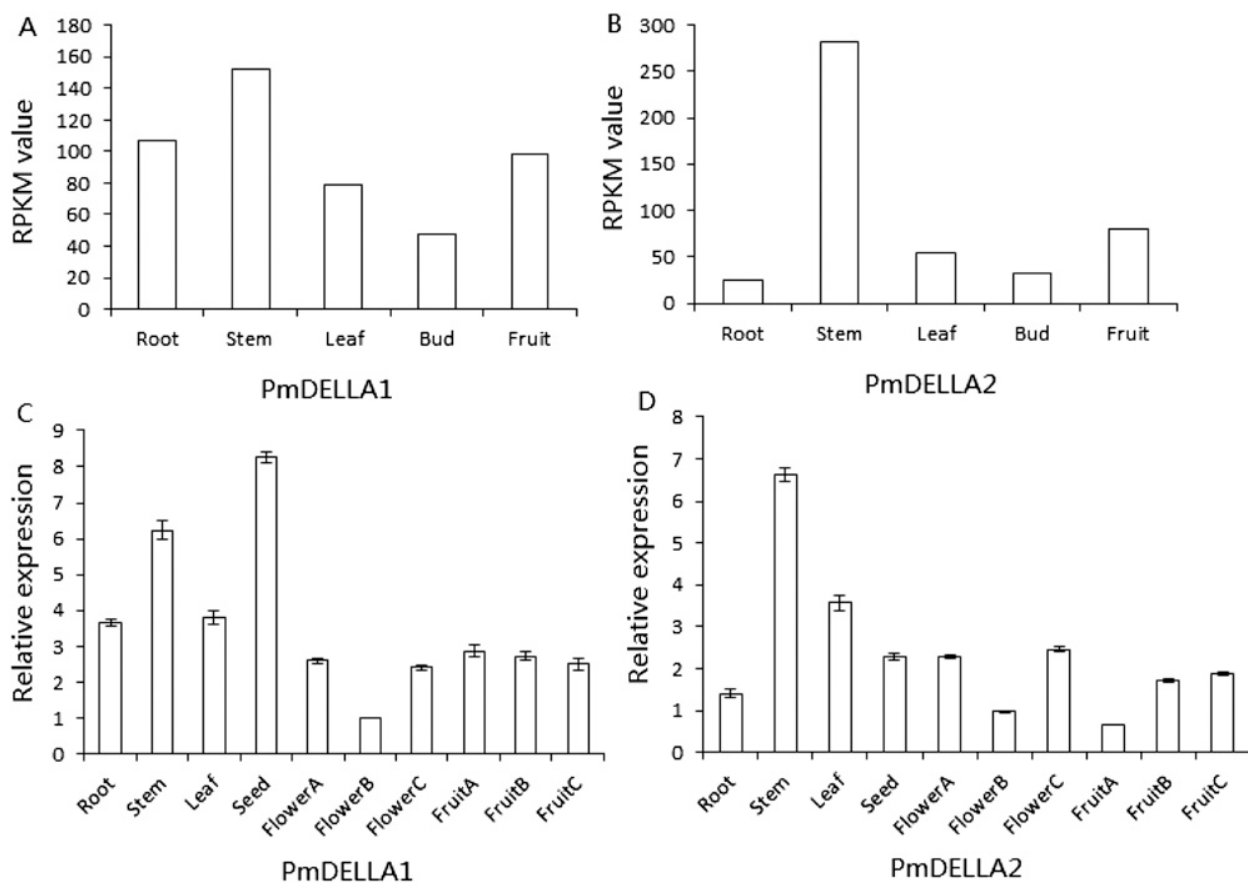

Fig. 4. Expression levels of (A) PmDELLA1 and (B) PmDELLA2 in different organs of Prunus mume were tested by RNA sequencing. The values on the Y-axis represent the reads per kilobase of exon model per million mapped reads (RPKM) values. Roots were harvested from 2-year-old plants. Stem tissue was obtained from a young stem tip. New-sprouting young leaves were collected in the spring. Fruit was obtained after 5 weeks of blooming. Relative expressions of (C) PmDELLA1 and (D) PmDELLA2 in different organs and development stages of $P$. mume were tested by quantitative PCR. RNA was isolated from the root, stem and leaf of 2-year-old plants. Seeds were collected from mature fruits. Flowers and fruits were collected at the three developmental stages. Flower A, B, and C correspond to bud, full-bloom stage, and end-bloom stage and fruit A, B, and C correspond to 10,45 , and $90 \mathrm{~d}$ after blooming. The relative quantification values were calculated using two reference genes, the protein phosphatase 2A gene (PP2A) and ubiquitin-conjugating enzyme E2 (UBC). The average value with standard errors of the three repetitions was used to draw the figure.

was the monocotyledonous group that contained six DELLAs from five species. Groups II and III were dicotyledonous groups. PmDELLA1 and PmDELLA2 were clustered into Groups II and III, respectively. PmDELLA1 was grouped with AtGAI and AtRGA, while PmDELLA2 was grouped with AtRGL1-3. Group IV included DELLA-like proteins from moss and gymnosperm species. The phylogenetic tree also showed that PmDELLA1 is closely related to MdRGL1 and MhGAI1, while PmDELLA2 is closely related to MdRGL2 and MhDELLA.

EXPRESSION PATTERNS OF PmDELLAs in Different organs, AND AT VARIOUS FLOWER AND FRUIT DEVELOPMENTAL STAGES. To investigate the roles of PmDELLAs in different organs, and in flower and fruit development stages of mei, the expression patterns of PmDELLA1 and PmDELLA2 were determined by RNA-seq and quantitative PCR.

The expression of PmDELLAs in five mei organs was analyzed using RNA-Seq data. We extracted the transcriptomic data for five organs (root, stem, leaf, bud, and fruit). Reads per kilobase of exon model per million mapped reads (RPKM) values of the genes in the five organs

which was differentiated since the divination of Plantae and Ptotistae.

The number of the DELLA in mei was equal to that of grape (2) and exceeded that of strawberry (1), barrel medic (1), tomato (1), rice (1), spikemoss (1), and bryophyte (1). It was, however, less than that of apple (5), cucumber (3), black cottonwood (6), arabidopsis (5), chinese cabbage (5), orange (3), cocoa (3), maize (3), and banana (3). The genome size of mei $(280.1 \mathrm{Mb})$ was greater than that of cucumber $(203.0 \mathrm{Mb})$, barrel medic $(257.6 \mathrm{Mb})$, and arabidopsis $(135.0 \mathrm{Mb})$, but less than that of the other species examined. The number of DELLA had no direct relationship with the genome size and showed no clear pattern between dicotyledons and monocotyledons, which was the same for woody and herbal plants. We also found that apple, black cottonwood, arabidopsis, and chinese cabbage had higher numbers of DELLAs. All four of these genomes underwent a recent whole-genome duplication (WGD) (Song et al., 2014; Tuskan et al., 2006; Velasco et al., 2010). However, mei has not undergone a recent WGD, which is the same as strawberry and grape (Jaillon et al., 2007; Shulaev et al., 2011). Thus, WGD events facilitated the expansion of the DELLA subfamily.

To investigate the phylogenetic relationship between PmDELLAs and other DELLA proteins, 41 DELLA protein sequences (Supplemental Table 1) from 22 species were downloaded from the NCBI to construct a phylogenetic tree (Fig. 3). The phylogenetic tree included four groups. Group I are shown in Figure 4A and B. Both genes were expressed in all five organs, with the highest expression levels in the stem. The expression level of PmDELLA1 in the five organs ranged from 48 to 152 (RPKM values), whereas the expression level of PmDELLA2 ranged from 25 to 281 . The different expression profiles of PmDELLAs indicated that they may have similar functions in some organs and specific functions in others.

The expressions of the PmDELLAs in seeds and during flower and fruit development stages were analyzed by quantitative PCR. As shown in Figure 4C and D, both genes are actively transcribed, although their expression patterns are different. Both genes showed high expression in the stem, which was consistent with the RNA-seq result. We speculated that they may have important functions in stem development. PmDELLA1 showed very high expression in the seed; whereas, PmDELLA2 showed very low expression. This indicated that PmDELLA1 may have more important functions in seed germination than PmDELLA2. In addition, the two genes showed the same expressions patterns during floral blooming. They were both down-regulated in flowers at stage $B$ and slightly up-regulated at stage $C$. This suggested that they have similar functions during floral blooming. By contrast, the two genes showed different patterns during fruit development. The expression level of PmDELLA1 gradually increased during fruit development, whereas PmDELLA2 expression decreased stage by stage. This suggested that they play different roles in fruit development. 
A

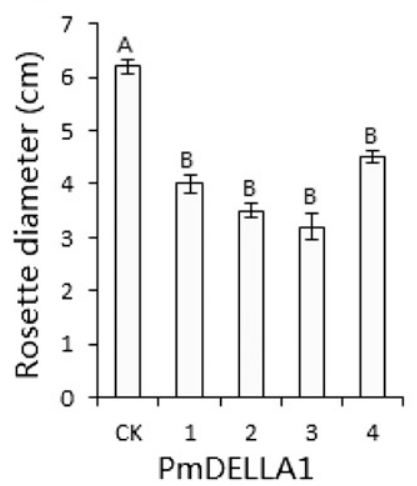

B

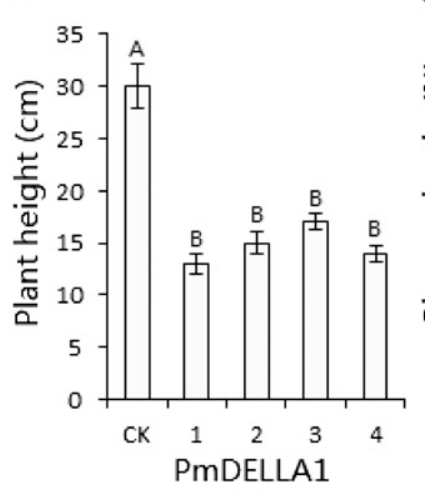

$\mathrm{C}$

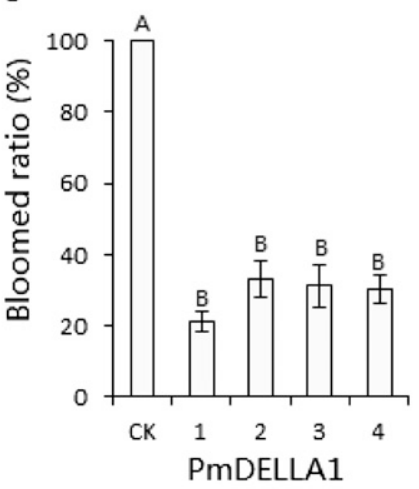

$E$
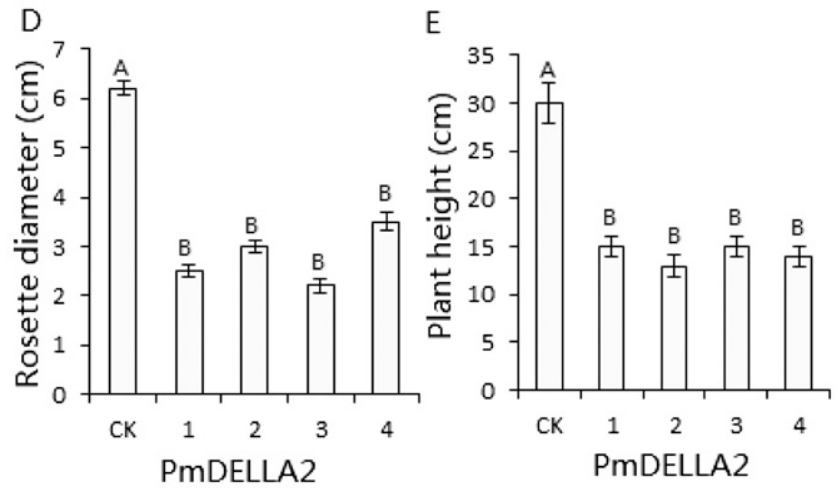

$\mathrm{F}$

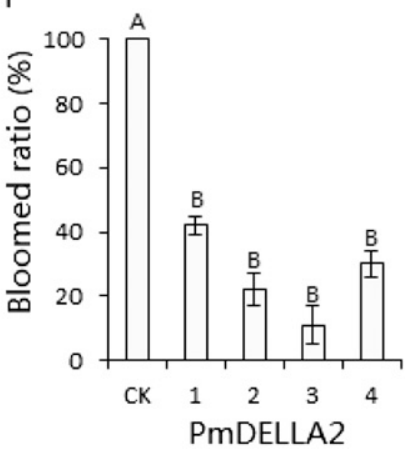

$\mathrm{H}$

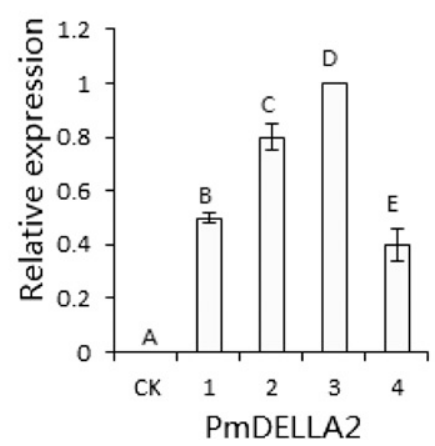

Fig. 5. Rosette diameters, plant height, and bloomed ratio of (A-C) PmDELLA1 and (D-F) PmDELLA2 transgenic Arabidopsis thaliana lines (four lines per gene labeling 1-4) and wild-type control plants (CK) are shown. Relative expressions of PmDELLA1 (G) and PmDELLA2 (H) transgenic A. thaliana lines and CK were determined by quantitative PCR. For measuring rosette diameters and plant height, plants were cultured under long-day conditions ( $16 \mathrm{~h}$ light $/ 8 \mathrm{~h}$ dark) for $25 \mathrm{~d}$. For recording the bloomed ratio, plants were cultured under short-day conditions $(8 \mathrm{~h}$ light $/ 16 \mathrm{~h}$ dark) for $60 \mathrm{~d}$. Each treatment was conducted in three repetitions. Data are collected from 20 plants per line for each repetition. Different letters indicate that $\mathrm{CK}$ and transgenic lines differ significantly from each other by Student-Newman-Keuls test $($ alpha $=0.01)$. Values shown are means with standard errors.

Phenotype analysis of transgenic arabidopsis overexpressing PuDELLAs. Binary vectors containing the full-length PmDELLA1 or PmDELLA2 cDNAs sequences were transformed into arabidopsis using the floral dip method (Clough and Bent, 1998) for observing the phenotype of transformed arabidopsis. Four independent 35S:PmDELLA1 and 35S:PmDELLA2 transgenic lines were generated. Compared with CK, four lines overexpressing PmDELLA1 showed $27 \%$ to $48 \%$ smaller in rosette diameter (Fig. $5 \mathrm{~A}$ ), $43 \%$ to $56 \%$

shorter in plant height (Fig. 5B), and $65 \%$ to $79 \%$ decrease in bloomed ratio (Fig. 5C), while four lines overexpressing PmDELLA2 showed $43 \%$ to $50 \%$ shorter in plant height (Fig. 5D), $43 \%$ to $64 \%$ smaller in rosette diameter (Fig. 5E), and 58\% to $89 \%$ decrease in bloomed ratio (Fig. 5F). Significant analysis showed that CK and transgenic lines had significant difference by StudentNewman-Keuls test $($ alpha $=0.01)$ among rosette diameter, plant height and bloomed ratio. Transgene expression levels of PmDELLAs in each line were determined by quantitative PCR (Fig. 5G and $\mathrm{H}$ ).

Transgenic plants overexpression of genes encoding DELLA proteins has been shown to reduce the response to exogenous GA (Ait Ali et al., 2003; Fleck and Harberd, 2002; Fu et al., 2001; Itoh et al., 2002). We observed that $\mathrm{CK}$ on $\mathrm{GA}_{3}$ media increased $23 \%$ in seedling hypocotyl elongation compared with CK on normal media. Four PmDELLA1 lines on $\mathrm{GA}_{3}$ media increased $9 \%$ to $25 \%$ compared with four lines on normal media, while four PmDELLA2 lines increased $10 \%$ to $15 \%$ on $\mathrm{GA}_{3}$ media compared with them on normal media (Fig. 6A and B). We observed $\mathrm{CK}$ and transgenic lines all had response to exogenous $\mathrm{GA}_{3}$. However, significant analysis (data not shown) showed that the difference values of $\mathrm{CK}$ on $\mathrm{GA}_{3}$ media and that on normal media and the difference values of transgenic lines of PmDELLAs on $\mathrm{GA}_{3}$ media and that on normal media had significant difference by Student-NewmanKeuls test (alpha $=0.01)$. This indicated that transgenic arabidopsis overexpressing PmDELLAs reduced the response to exogenous $\mathrm{GA}_{3}$.

\section{Discussion}

Mei is a woody perennial tree with an intergenerational in a cycle of 3-5 years (Chen, 1996). Prunus crops are economically important because of their tasty fruit, which provide unique dietary contributions to consumers, and as ornamental plants, especially as dwarf cultivars as indoor plants. For these valuable characters, breeders have used many ways to control flowering, fruit growth, and canopy architecture. In this study, two PmDELLAs encoding negative regulators of the GA signaling pathway were identified in the mei genome. Both genes had two signature motifs, DELLA and TVHYNP, which define the 


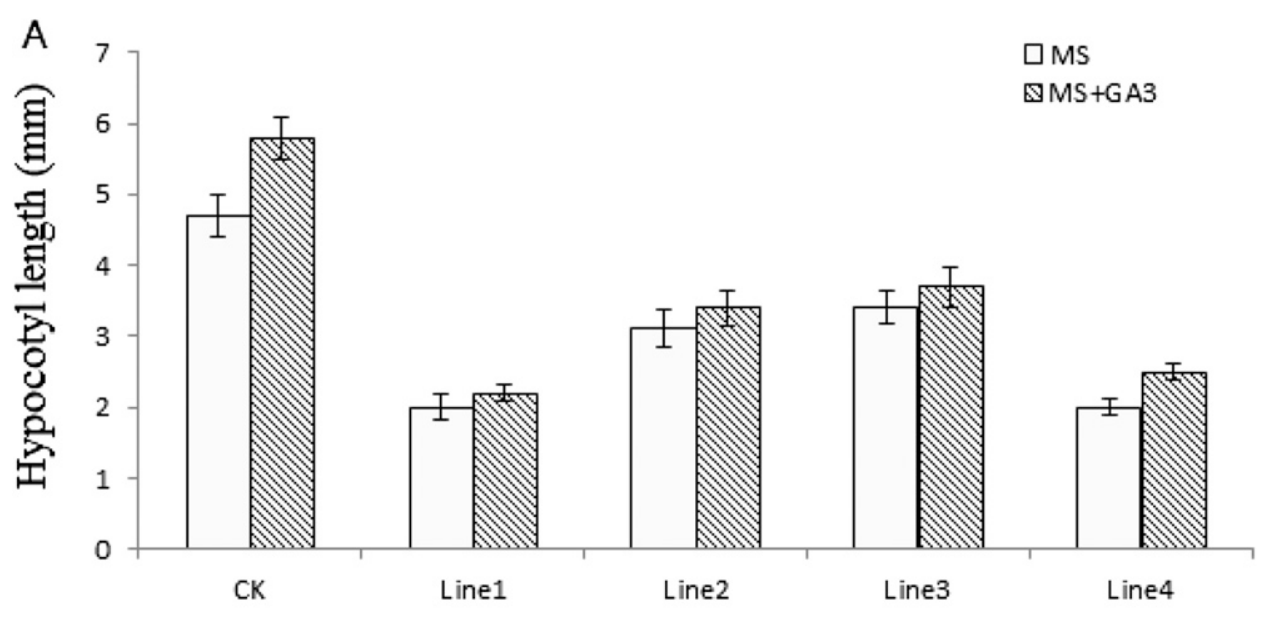

PmDELLA1

B

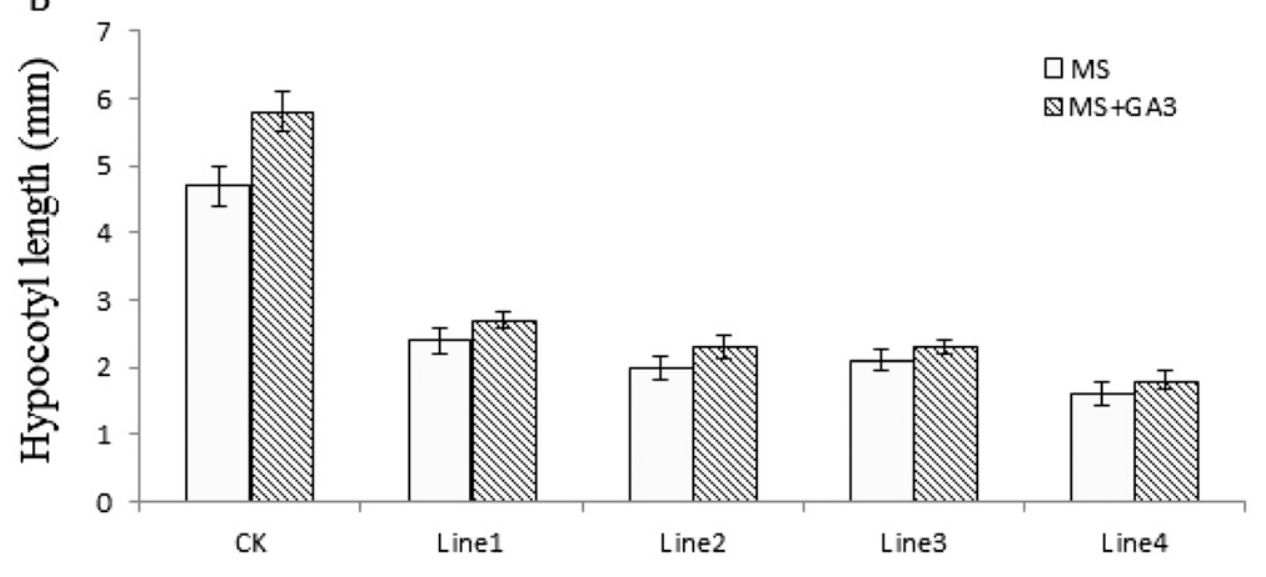

PmDELLA2

Fig. 6. Hypocotyl length of (A) PmDELLA1 and (B) PmDELLA2 transgenic Arabidopsis thaliana lines (four lines per gene) and wild-type control plants (CK) on $\mathrm{GA}_{3}$ media (oblique lines) and normal media (blank). Seeds were germinated on $0.5 \times$ Murashige and Skoog culture medium (MS) in the presence or absence of $10^{-4} \mathrm{M} \mathrm{GA}_{3}$ and grown in culture room $(16 \mathrm{~h}$ light $/ 8 \mathrm{~h}$ dark) for $7 \mathrm{~d}$. Data are collected from 25 seedlings per line for each repetition (totally three repetitions). Mean hypocotyl lengths are shown with standard errors as vertical bars.

(group I) and dicots (groups II and III) were divided into two independent groups. This indicated that DELLA proteins were diversified after the monocot-dicot split. We could not predict the evolution of DELLAs any earlier, because there is little information for moss and gymnosperms species. We observed that PmDELLA1 and PmDELLA2 were clustered into Groups II and III, respectively. A previous study showed that GAI and RGA function to control cell expansion in the hypocotyl, shoot, and root, while RGL2 is the major DELLA protein regulating germination. Expression analysis showed that PmDELLA2 showed very high expression levels in seed. All this findings indicated that PmDELLA2 probably have different functions with PmDELLA1. Although our preliminary analysis of the known DELLA genes supported this hypothesis, the amount of complete genome data for plants is insufficient to adequately measure the evolutionary history of the DELLA gene.

Expression analysis of PmDELLAs in different organs showed that both PmDELLAs were active in all organs. Previous studies showed that organ specificities of DELLA genes were different among species. $S L R$ and $S L N$ are preferentially expressed in certain organs (Chandler et al., 2002; Ogawa et al., 2000). By contrast, $R G A$ and $G A I$ are expressed constitutively in all tissues and appear to be regulated primarily by proteolysis (Fu et al.,

DELLA subfamily and are necessary for GA-induced degradation of DELLA proteins (Foster et al., 2007; Pysh et al., 1999). Mutation of either of these domains results in a semidominant, GA-insensitive, dwarfed phenotype in diverse plant species.

With the abundance of genome resources, the information stored in various genomes can be explored to elucidate the mechanisms that regulate the growth and development of species. Comparative analysis showed that the expansion of the DELLA subfamily is related to genome duplication events. As a key subfamily of GRAS proteins, members of the DELLA subfamily are involved in regulating the GA response. GAs are important hormones that regulate plant development. In woody plants, GAs help to reduce bud dominance, promote flowering, and increase fruit setting. We speculated that some DELLA genes have been retained after the genome duplication process to facilitate the adaption of woody plants to complex environments. We believe that the DELLA subfamily plays an important role in higher plant growth and development.

Phylogenetic analysis showed that 41 DELLA protein sequences could be classed into four groups. The monocots
2004; Lee et al., 2002; Tyler et al., 2004). Only RGL1, RGL2, and $R G L 3$ appear to be regulated at the transcript level (Lee et al., 2002; Tyler et al., 2004; Wen and Chang, 2002). We observed that both PmDELLAs appear to be transcriptionally regulated. However, the expression patterns of PmDELLAs in different organs varied. The two PmDELLAs showed different expression levels in seeds: very high for PmDELLA1 and very low for PmDELLA2. Previous studies showed that in arabidopsis, $R G L 1, R G L 2$, and $R G L 3$ are expressed at high levels only in germinating seeds, and/or flowers, and siliques (Lee et al., 2002; Tyler et al., 2004). Thus, the expression pattern of PmDELLA1 was consistent with genes encoding DELLA proteins in arabidopsis. In addition, unlike arabidopsis seeds, mei seeds require several weeks of low temperature $\left(0-4{ }^{\circ} \mathrm{C}\right)$ to germinate (Chen, 1996). Thus, we speculated that the germination of mei seeds might be regulated more by environmental and physiological factors than by the activity of the PmDELLAs.

The expressions of PmDELLAs at various flower and fruit development stages showed that both PmDELLAs had the same 
patterns during floral blooming, but different patterns during fruit development. Both genes were expressed at relatively low levels during flower blooming. Previous studies showed that GA promotes flowering by targeting DELLA proteins for destruction in arabidopsis, which ultimately leads to activation of $L E A F Y$, a key floral meristem identity gene (Achard et al., 2004; Greene, 1989). Certain GA responses such as internode elongation, leaf expansion, and seed germination are conserved between species, whereas the relationship between GA and flowering is not. Changes in the relationships between key regulatory proteins can lead to rapid morphological evolution (Doebley and Lukens, 1998; Purugganan, 2000). In woody plants, specific GA species repress flowering, particularly in grape and apple (Greene, 1989). We speculated that PmDELLAs might be positive regulators of flowering through releasing the repression of GA during floral blooming.

Transgenic arabidopsis lines expressing PmDELLAs showed a reduction in leaf size and stem height, and delayed flowering. Compared with the controls, the transgenic seedlings had shorter hypocotyls and exhibited reduced elongation responses to exogenous GA. Previous studies showed that overexpression of DELLA proteins in arabidopsis, rice, and tobacco (Nicotiana tabacum) caused a range of dwarfed phenotypes that varied according to the transgene expression level (Itoh et al., 2002). The dwarfed phenotypes and the increases hypocotyls of transgenic lines of PmDELLAs revealed that the two $P m D E L L A s$ probably function as negative regulators of GA-dependent processes in mei.

In summary, we studied DELLA subfamily in mei and 19 other species, including seven woody plants. We deduced that $P m D E L L A s$ are functionally conserved in mei as the negative transcription regulator of GA. The identification of PmDELLAs will provide a molecular basis for the genetic improvement of mei, especially for producing dwarf cultivars and regulating flowering time in woody plant. Our bioinformatics and functional analysis provides valuable information to improve the economic, agronomic, and ecological properties of mei and other Rosaceae fruit trees.

\section{Literature Cited}

Achard, P., A. Herr, D.C. Baulcombe, and N.P. Harberd. 2004. Modulation of floral development by a gibberellin-regulated microRNA. Development 131:3357-3365.

Ait Ali, T., C. Randsand, and N.P. Harberd. 2003. Flexible control of plant architecture and yield via switchable expression of arabidopsis gai. Plant Biotechnol. J. 1:337-343.

Artimo, P., M. Jonnalagedda, K. Arnold, D. Baratin, G. Csardi, E. De Castro, S. Duvaud, V. Flegel, A. Fortier, and E. Gasteiger. 2012. ExPASy: SIB bioinformatics resource portal. Nucleic Acids Res. 40: W597-W603.

Boss, P.K. and M.R. Thomas. 2002. Association of dwarfism and floral induction with a grape 'green revolution' mutation. Nature 416:847-850.

Cao, D., A. Hussain, H. Cheng, and J. Peng. 2005. Loss of function of four DELLA genes leads to light and gibberellin-independent seed germination in arabidopsis. Planta 223:105-113.

Chandler, P.M., A. Marion-Poll, M. Ellis, and F. Gubler. 2002. Mutants at the Slenderl locus of barley cv Himalaya. Molecular and physiological characterization. Plant Physiol. 129:181-190.

Chen, J.Y. 1996. Chinese mei flowers. (in Chinese) Hainan Publ. House, Haikou, China.

Cheng, H., L. Qin, S. Lee, X. Fu, D.E. Richards, D. Cao, D. Luo, N.P. Harberd, and J. Peng. 2004. Gibberellin regulates arabidopsis floral development via suppression of DELLA protein function. Development 131:1055-1064.
Clough, S.J. and A.F. Bent. 1998. Floral dip: A simplified method for agrobacterium-mediated transformation of Arabidopsis thaliana. Plant J. 16:735-743.

D'Hont, A., F. Denoeud, J. Aury, F. Baurens, F. Carreel, O. Garsmeur, B. Noel, S. Bocs, G. Droc, and M. Rouard. 2012. The banana (Musa acuminata) genome and the evolution of monocotyledonous plants. Nature 488:213-217.

Davies, P.J. 1995. Plant hormones: Physiology, biochemistry, and molecular biology. Kluwer Acadademoc Publ., Dordrecht, The Netherlands.

Dill, A. and T. Sun. 2001. Synergistic derepression of gibberellin signaling by removing $R G A$ and GAI function in Arabidopsis thaliana. Genetics 159:777-785.

Doebley, J. and L. Lukens. 1998. Transcriptional regulators and the evolution of plant form. Plant Cell 10:1075-1082.

Finn, R.D., A. Bateman, J. Clements, P. Coggill, R.Y. Eberhardt, S.R. Eddy, A. Heger, K. Hetherington, L. Holm, and J. Mistry. 2013. Pfam: The protein families database. Nucleic Acids Res., doi: 10.1093/nar/gkt1223.

Fleck, B. and N.P. Harberd. 2002. Evidence that the arabidopsis nuclear gibberellin signaling protein GAI is not destabilized by gibberellin. Plant J. 32:935-947.

Foster, T., C. Kirk, W.T. Jones, A.C. Allan, R. Espley, S. Karunairetnam, and J. Rakonjac. 2007. Characterization of the DELLA subfamily in apple (Malus x domestica Borkh.). Tree Genet. Genomes 3:187-197.

Fu, X., D. Sudhakar, J. Peng, D.E. Richards, P. Christou, and N.P. Harberd. 2001. Expression of arabidopsis $G A I$ in transgenic rice represses multiple gibberellin responses. Plant Cell 13:1791-1802.

Fu, X., D.E. Richards, B. Fleck, D. Xie, N. Burton, and N.P. Harberd. 2004. The arabidopsis mutant sleepyl (gar2-1) protein promotes plant growth by increasing the affinity of the SCF SLY1 E3 ubiquitin ligase for DELLA protein substrates. Plant Cell 16:1406-1418.

Gallego-Bartolomé, J., E.G. Minguet, J.A. Marín, S. Prat, M.A. Blázquez, and D. Alabadí. 2010. Transcriptional diversification and functional conservation between DELLA proteins in arabidopsis. Mol. Biol. Evol. 27:1247-1256.

Goodstein, D.M., S. Shu, R. Howson, R. Neupane, R.D. Hayes, J. Fazo, T. Mitros, W. Dirks, U. Hellsten, and N. Putnam. 2012. Phytozome: A comparative platform for green plant genomics. Nucleic Acids Res. 40:D1178-D1186.

Greene, D.W. 1989. Gibberellins $\mathrm{A}_{4+7}$ influence fruit set, fruit quality, and return bloom of apples. J. Amer. Soc. Hort. Sci. 6:1238-1240.

Itoh, H., M. Ueguchi-Tanaka, Y. Sato, M. Ashikari, and M. Matsuoka. 2002. The gibberellin signaling pathway is regulated by the appearance and disappearance of SLENDER RICE1 in nuclei. Plant Cell 14:57-70.

Jaillon, O., J. Aury, B. Noel, A. Policriti, C. Clepet, A. Casagrande, N. Choisne, S. Aubourg, N. Vitulo, and C. Jubin. 2007. The grapevine genome sequence suggests ancestral hexaploidization in major angiosperm phyla. Nature 449:463-467.

Jones, P., D. Binns, H. Chang, M. Fraser, W. Li, C. McAnulla, H. McWilliam, J. Maslen, A. Mitchell, and G. Nuka. 2014. InterProScan 5: Genome-scale protein function classification. Bioinformatics 30:1236-1240.

Larkin, M.A., G. Blackshields, N.P. Brown, R. Chenna, P.A. McGettigan, H. McWilliam, F. Valentin, I.M. Wallace, A. Wilm, and R. Lopez. 2007. Clustal W and Clustal X version 2.0. Bioinformatics 23:29472948.

Lee, S., H. Cheng, K.E. King, W. Wang, Y. He, A. Hussain, J. Lo, N.P. Harberd, and J. Peng. 2002. Gibberellin regulates arabidopsis seed germination via $R G L 2$, a GAI/RGA-like gene whose expression is upregulated following imbibition. Genes Dev. 16:646-658.

Locascio, A., M.A. Blázquezand, and D. Alabadí. 2013. Genomic analysis of DELLA protein activity. Plant Cell Physiol. 54:12291237.

Looney, N.E. 1997. Hormones and horticulture. HortScience 32:10141018. 
Ogawa, M., T. Kusano, M. Katsumi, and H. Sano. 2000. Rice gibberellin-insensitive gene homolog, OsGAI, encodes a nuclearlocalized protein capable of gene activation at transcriptional level. Gene 245:21-29.

Peng, J., D.E. Richards, N.M. Hartley, G.P. Murphy, K.M. Devos, J.E. Flintham, J. Beales, L.J. Fish, A.J. Worland, and F. Pelica. 1999. 'Green revolution' genes encode mutant gibberellin response modulators. Nature 400:256-261.

Peng, J., P. Carol, D.E. Richards, K.E. King, R.J. Cowling, G.P. Murphy, and N.P. Harberd. 1997. The arabidopsis GAI gene defines a signaling pathway that negatively regulates gibberellin responses. Genes Dev. 11:3194-3205.

Purugganan, M.D. 2000. The molecular population genetics of regulatory genes. Mol. Ecol. 9:1451-1461.

Pysh, L.D., J.W. Wysocka Diller, C. Camilleri, D. Bouchez, and P.N. Benfey. 1999. The GRAS gene family in arabidopsis: Sequence characterization and basic expression analysis of the scarecrow-like genes. Plant J. 18:111-119.

Rademacher, W. 2000. Growth retardants: Effects on gibberellin biosynthesis and other metabolic pathways. Annu. Rev. Plant Physiol. Plant Mol. Biol. 51:501-531.

Rhee, S.Y., W. Beavis, T.Z. Berardini, G. Chen, D. Dixon, A. Doyle, M. Garcia-Hernandez, E. Huala, G. Lander, and M. Montoya. 2003. The arabidopsis information resource (TAIR): A model organism database providing a centralized, curated gateway to arabidopsis biology, research materials and community. Nucleic Acids Res. 31:224-228.

Shulaev, V., D.J. Sargent, R.N. Crowhurst, T.C. Mockler, O. Folkerts, A.L. Delcher, P. Jaiswal, K. Mockaitis, A. Liston, and S.P. Mane. 2011. The genome of woodland strawberry (Fragaria vesca). Nat. Genet. 43:109-116.

Silverstone, A.L., C.N. Ciampaglio, and T. Sun. 1998. The arabidopsis $R G A$ gene encodes a transcriptional regulator repressing the gibberellin signal transduction pathway. Plant Cell 10:155-169.

Song, X., T. Liu, W. Duan, Q. Ma, J. Ren, Z. Wang, Y. Li, and X. Hou. 2014. Genome-wide analysis of the GRAS gene family in chinese cabbage (Brassica rapa ssp. pekinensis). Genomics 103:135-146.

Tamura, K., D. Peterson, N. Peterson, G. Stecher, M. Nei, and S. Kumar. 2011. MEGA5: Molecular evolutionary genetics analysis using maximum likelihood, evolutionary distance, and maximum parsimony methods. Mol. Biol. Evol. 28:2731-2739.

Tian, C., P. Wan, S. Sun, J. Li, and M. Chen. 2004. Genome-wide analysis of the GRAS gene family in rice and arabidopsis. Plant Mol. Biol. 54:519-532.

Tuskan, G.A., S. Difazio, S. Jansson, J. Bohlmann, I. Grigoriev, U. Hellsten, N. Putnam, S. Ralph, S. Rombauts, and A. Salamov. 2006. The genome of black cottonwood, Populus trichocarpa (Torr. \& Gray). Science 313:1596-1604.

Tyler, L., S.G. Thomas, J. Hu, A. Dill, J.M. Alonso, J.R. Ecker, and T. Sun. 2004. DELLA proteins and gibberellin-regulated seed germination and floral development in arabidopsis. Plant Physiol. 135:1008-1019.

Vandesompele, J., D.P. Katleen, P. Filip, P. Bruce, V.R. Nadine, A.D. Paepe, and S. Frank. 2002. Accurate normalization of real-time quantitative RT-PCR data by genometric averaging of multiple internal control genes. Gen. Biol. doi: $10.1186 / \mathrm{gb}-2002-3-7$ research0034.

Velasco, R., A. Zharkikh, J. Affourtit, A. Dhingra, A. Cestaro, A. Kalyanaraman, P. Fontana, S.K. Bhatnagar, M. Troggio, and D. Pruss. 2010. The genome of the domesticated apple (Malus $\times$ domestica Borkh.). Nat. Genet. 42:833-839.

Wang, H., J. Chen, Q. Tian, S. Wang, X. Xia, and W. Yin. 2014a. Identification and validation of reference genes for Populous euphratica gene expression analysis during abiotic stresses by quantitative real-time PCR. Physiol. Plant. 152:529-545.

Wang, T., R. Hao, H. Pan, T. Cheng, and Q. Zhang. 2014b. Selection of suitable reference genes for quantitative real-time polymerase chain reaction in Prunus mume during flowering stages and under different abiotic stress conditions. J. Amer. Soc. Hort. Sci. 139:113-122.

Wen, C. and C. Chang. 2002. Arabidopsis RGL1 encodes a negative regulator of gibberellin responses. Plant Cell 14:87-100.

Zhang, D., L.M. Iyerand, and L. Aravind. 2012a. Bacterial GRAS domain proteins throw new light on gibberellic acid response mechanisms. Bioinformatics 28:2407-2411.

Zhang, Q., W. Chen, L. Sun, F. Zhao, B. Huang, W. Yang, Y. Tao, J. Wang, Z. Yuan, and G. Fan. 2012b. The genome of Prunus mume. Nat. Commun. 3:1318. 
Supplemental Table 1. Accession numbers of genes from NCBI database used for phylogenetic analyses.

\begin{tabular}{|c|c|c|}
\hline Gene & NCBI accession no. & Species \\
\hline AtGAI & CAA75492 & Arabidopsis thaliana \\
\hline AtRGA & CAA72177 & A. thaliana \\
\hline AtRGL1 & NP_176809 & A. thaliana \\
\hline AtRGL2 & NP_186995 & A. thaliana \\
\hline AtRGL3 & NP_197251 & A. thaliana \\
\hline BrRGA2 & Q5BN23 & Brassica rapa \\
\hline BrRGA1 & Q5BN22 & B. rapa \\
\hline CmGAIP & Q6EI06 & Cucurbita maxima \\
\hline CmGAIP-B & Q6EI05 & C. maxima \\
\hline GhGAI & Q84TQ7 & Gossypium hirsutum \\
\hline GhRGA & AAY28970 & G. hirsutum \\
\hline LsDELLA1 & BAG71200 & Lactuca sativa \\
\hline LsDELLA2 & BAG71201 & L. sativa \\
\hline LeGAI & Q7Y1B6 & Solanum lycopersicum \\
\hline MdRGL1 & AAY56752 & Malus $\times$ domestica \\
\hline MdRGL2 & AAY56750 & M. $\times$ domestica \\
\hline MhDELLA & ABS50250 & Malus hupehensis \\
\hline MhGAI1 & ABL61270 & M. hupehensis \\
\hline OsSLR1 & NP_001051032 & Oryza sativa \\
\hline PvDELLA1 & BAF62636 & Phaseolus vulgaris \\
\hline PvDELLA2 & BAF62637 & P. vulgaris \\
\hline PpDELLA1 & XM_001774262 & Physcomitrella patens \\
\hline PpDELLA2 & XM_001754038 & P. patens \\
\hline PsCRY & $\mathrm{ABI} 34432$ & Pisum sativum \\
\hline PsDELLA & ABI30654 & P. sativum \\
\hline PtDELLA1 & XM_002312414 & Populus trichocarpa \\
\hline PtDELLA2 & XM_002314763 & P. trichocarpa \\
\hline PtDELLA3 & XM_001774262 & P. trichocarpa \\
\hline PtDELLA4 & XM_001754038 & P. trichocarpa \\
\hline RcGAI & XP_002534030 & Ricinus communis \\
\hline RcGAIP-B & XP_002527794 & R. communis \\
\hline SoGAI & AAZ08571 & Saccharum officinarum \\
\hline SkDELLA & ABU63412 & Selaginella kraussiana \\
\hline SmDELLA & ABX10758 & Selaginella moellendorffii \\
\hline SbDELLA & XP_002466594 & Sorghum bicolor \\
\hline SpDELLA & ABU63411 & Sphagnum palustre \\
\hline TaRhtD1a & Q9ST59 & Triticum aestivum \\
\hline VvDELLA1 & XM_002534030 & Vitis vinifera \\
\hline VvGAI1 & XM_002284612 & $V$. vinifera \\
\hline $\mathrm{ZmD8}$ & Q9ST48 & Zea mays \\
\hline ZmD9 & ABI84225 & Z. mays \\
\hline
\end{tabular}

Supplemental Table 2. Primers used for clone and quantitive PCR expression.

\begin{tabular}{llll}
\hline Type & Gene & \multicolumn{1}{c}{ Forward primer } & Reverse primer \\
\hline Clone & PmDELLA1 & ATGAAAAGAGAGCATCAGATTTATCATCAATATCCC & TCAGTGAGCCATCACCGAGTCGCA \\
& PmDELLA2 & ATGAAGAGAGATCACCGGGATACCTG & TCACCGGGTTGACTCAGTCGAAC \\
Quantitive PCR & PmDELLA1 & TTTGGCAGATCTCGAACCCT & ACAAGACCTTTTCAACCGCC \\
& PmDELLA2 & GAGATCAACCCACCTCCCAA & GTTGGACTTGTTGCTGCTGA \\
& PP2A & ATATAGCTGCTCAGTTCAACC & AAAAACAGTCACCACATTCTT \\
& UBC & GCAGGTGGAGTGTTCCTTGTG & GGCAGGGCTCCACTGTTCTTT \\
\hline
\end{tabular}

